\title{
Atividades para Melhoria da Adesão à TARV em Serviços de Saúde do SUS no Estado de São Paulo, 2007'
}

\section{Interventions to Enhance Haart Adherence in HIV/Aids Health Services in the State of São Paulo, Brazil, 2007}

\author{
Joselita Maria de Magalhães Caraciolo \\ Médica. Departamento de Medicina Preventiva - FM/USP - Equipe \\ QUALIAIDS. Centro de Referência e Treinamento DST/Aids - SES/SP. \\ Endereço: Rua Santa Cruz, 8ı, Vila Mariana, CEP 04121-000, São \\ Paulo, SP, Brasil.

\section{Ernani Tiaraju de Santa Helena} \\ Doutorado. Departamento de Medicina - Universidade Regional \\ de Blumenau. Departamento de Medicina Preventiva - FM/USP \\ - Equipe QUALIAIDS. \\ Endereço: Rua Antonio da Veiga, 140, Victor Konder, Caixa-Postal \\ 1507, CEP 89010-971, Blumenau, SC, Brasil.
}

\section{Caritas Relva Basso}

Mestrado. Departamento de Medicina Preventiva - FM/USP - Equipe QUALIAIDS. Centro de Referência e Treinamento DST/Aids - SES/SP.

Endereço: Rua Santa Cruz, 81, Vila Mariana, CEP 04121-000, São Paulo, SP, Brasil.

\section{Rosa Alencar de Souza}

Médica. Centro de Referência e Treinamento DST/Aids - SES/SP. Endereço: Rua Santa Cruz, 81, Vila Mariana, CEP 04121-000, São Paulo, SP, Brasil.

\section{Mariliza Henrique da Silva}

Médica. Centro de Referência e Treinamento DST/Aids - SES/SP.

Endereço: Rua Santa Cruz, 8I, Vila Mariana, CEP 04121-000, São Paulo, SP, Brasil.

\section{Valvina Madeira Adão}

Psicóloga. Centro de Referência e Treinamento DST/Aids - SES/SP. Endereço: Rua Santa Cruz, 81, Vila Mariana, CEP 04121-000, São Paulo, SP, Brasil.

\section{Marcos Túlio Raposo}

Universidade Estadual do Sudoeste da Bahia. Departamento de Medicina Preventiva - FM/USP - Equipe QUALIAIDS.

Endereço: José Moreira Sobrinho, S/N, Jequiezinho, CEP 45200-000, Jequié, BA, Brasil.

\section{Maria Inês Battistella Nemes}

Departamento de Medicina Preventiva - FM/USP - Equipe QUALIAIDS.

Endereço: Av. Dr. Arnaldo, 455, 2 andar, sala 2243, Cerqueira Cesar, CEP o1246-903, São Paulo, SP, Brasil.

E-mail: mibnemesळusp.br

I Projeto de pesquisa financiado com recursos FAPESP, CHAMADA

PP-SUS 2006/2007. Processo N²006/61277-6.

\section{Resumo}

O sucesso da terapia antirretroviral (TARV) para o tratamento da Aids depende da manutenção de altas taxas de adesão à terapêutica medicamentosa. Em que pese o destaque que o plano propositivo do Programa Nacional de DST/Aids tem dado para a questão, não se conhecem as tecnologias voltadas para a adesão em curso nos serviços. Com base na análise de questionário semiestruturado distribuído a todos os serviços ambulatoriais do Estado de São Paulo, este estudo descreve os tipos de intervenções implantados. No Estado de São Paulo, a adesão à TARV está incorporada nas atividades ambulatoriais dos serviços que assistem PVHA. Quase todos os serviços informam realizar atividades individuais e, a maioria, pelo menos uma atividade coletiva para melhorar a adesão. Entretanto, para grande parte dos serviços as atividades para a adesão, bem como a avaliação da adesão do paciente, ocorrem nas consultas médicas e de enfermagem. Isso indica que o trabalho de adesão consiste predominantemente de questionamentos e discussões com o paciente: o profissional decide de que modo e quando abordar a adesão. Os serviços especializados e que acompanham o maior número de pacientes tendem a apresentar atividades mais qualificadas e mais específicas.

Palavras-chave: Síndrome de Imunodeficiência Adquirida; Cooperação do Paciente; Pesquisa sobre Serviços de Saúde; Terapia Antirretroviral de Alta Atividade. 


\section{Abstract}

The success of antiretroviral therapy for AIDS treatment depends on the maintenance of high rates of adherence to drug therapy. Despite the emphasis given to the question by the Brazilian STD / AIDS Program, the adherence technologies that are in course on outpatient services are not known. Based on the analysis of a semi-structured questionnaire distributed to all outpatient services of the State of São Paulo, this study describes the types of interventions that have been implemented. In the State of São Paulo, adherence to HAART is incorporated into the activities of outpatient services. Almost all services inform interventions administered to individuals, and the majority, at least one group intervention to improve adherence. However, in many outpatient services, adherence interventions, as well as the assessment of patient's adherence, take place during nursing and medical consultations. This indicates that the adherence technology approach consists predominantly of questioning and discussions with the patient, meaning that the professionals decide when and how they address adherence. The larger and more specialized services tend to have more qualified and more specific activities.

Keywords: Acquired Immunodeficiency Syndrome; Patient Compliance; Health Services Research; Antiretroviral Therapy, Highly Active.

\section{Introdução}

O emprego da Terapia Antirretroviral (TARV) proporcionou dramático impacto na mortalidade por Aids e aumento na sobrevida. Entretanto, esse panorama depende da manutenção de altas taxas de adesão ao tratamento medicamentoso (Paterson e col., 200o). No Brasil, até dezembro de 2007, havia aproximadamente 180.640 pessoas sob TARV, das quais 67.234 no Estado de São Paulo (Brasil, 2008). A importância crucial da adesão ao tratamento tem sido reconhecida pelo Programa Nacional de DST/Aids desde o final dos anos 1990. Em que pese o destaque que o plano propositivo do Programa (Brasil, 2007) tem dado para a questão, não se conhecem as tecnologias voltadas para a adesão em curso nos serviços.

\section{Objetivo}

Este estudo descreve as atividades implantadas nos serviços do SUS, que atendem em nível ambulatorial pessoas vivendo com HIV/Aids no Estado de São Paulo, para a melhoria da adesão.

\section{Materiais e Métodos}

O universo do estudo foi constituído dos 179 serviços que constavam do banco de dados do Programa Estadual de HIV/Aids. Solicitou-se aos gerentes que respondessem um questionário semiestruturado com as variáveis: identificação e características gerais, parceria com Organização Não Governamental (ONG), número de adultos sob TARV, formas e frequência de avaliação da adesão, atividades em execução para melhoria da adesão, atividades para populações específicas e materiais utilizados.

Os dados foram digitados em banco de dados eletrônico (Epidata 3.o). São apresentadas as frequências absoluta e relativa com intervalos de $95 \%$ de confiança. Calcularam-se as medidas de tendência central e de dispersão. Para testar associação entre variáveis categóricas utilizou-se o teste de Qui-quadrado de Pearson, Exato de Fischer e Qui-quadrado de tendência linear, no nível de significância de $\mathrm{p}<0,05$.

Para explorar a interdependência das variáveis categóricas em espaço de menor dimensionalidade, utilizou-se da técnica de Análise de Correspondências Múltiplas. A proximidade dos pontos no mapa fatorial foi interpretada como similaridade de condições. 


\section{Resultados}

Responderam ao questionário 136 (76,0\%) serviços. Não há diferenças estatisticamente significantes entre os serviços que responderam e não responderam quando examinados sua distribuição por macroáreas do estado, número de pacientes em TARV e tipos de serviços (Ambulatório de DAT/AIDS, ambulatório de Moléstias Infecciosas, Ambulatório de Especialidades, Ambulatório de Hospitais, Unidades Básicas de Saúde).

A mediana de pessoas sob TARV foi de 133 (intervalo interquartílico de 50 a 397). As 17 Unidades Básicas de Saúde (12,8\% dos serviços) representam a maior proporção de serviços com menos de 20 pessoas, enquanto os 47 ambulatórios especializados em DST/Aids (35,3\%) e os 29 ambulatórios de MI (21,8\%) são representativos dos serviços maiores ( $\chi 2=75,3$ p<0,ooo1) (Gráfico 1).

\section{Gráfico I - Proporção do tamanho por tipo de serviço que atendem PVHA no estado de São Paulo $(n=131)$}

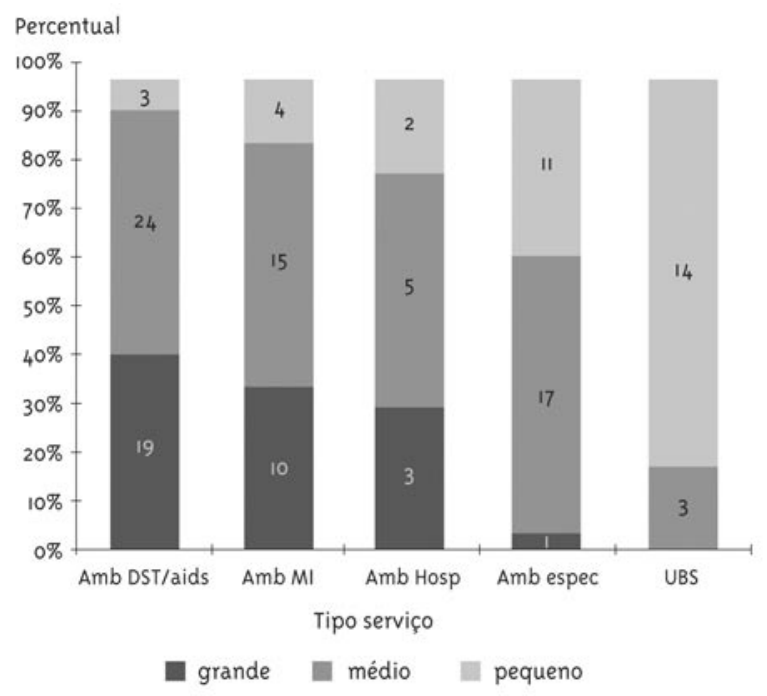

A grande maioria refere que a avaliação da adesão ocorre nas consultas médicas $(127 ; 93,4 \%)$ e de enfermagem (91; 66,9\%). Os serviços especializados em HIV/Aids informam maior proporção de avaliação por meio da retirada de medicamentos na farmácia do que os serviços de outros tipos $\left(\chi_{2}=12,0-p<0,05\right)$. 0 tamanho do serviço mostrou associação com o tipo de avaliação, isto é, naqueles que acompanham mais de 100 pessoas sob TARV cada profissional pergunta de uma maneira $\left(\chi_{2}=13,6-p<0,05\right)$.

A maioria das atividades individuais de estímulo à adesão é realizada por médicos (93,4\%) e enfermeiros
(67\%). Os serviços especializados em HIV/Aids referem maior proporção de envolvimento de psicólogos $\left(\chi_{2}=\right.$ $14,4-p<0,01)$ e assistentes sociais $\left(\chi_{2}=14,2-p<0,01\right)$ nessas atividades do que os demais tipos de serviço. Os serviços com maior número de pacientes também estão associados ao maior envolvimento de psicólogos $\left(\chi_{2}=21,5-p<0,01\right)$ e assistentes sociais $\left(\chi_{2}=19,0-p\right.$ $<\mathrm{O}, \mathrm{Ol})$.

Realizar atividades coletivas como grupos de adesão $\left(\chi_{2}=16,3-p<0,01\right)$, atividades em sala de espera $\left(\chi_{2}=10,8-p<0,05\right)$ e existência de outras atividades coletivas $\left(\chi_{2}=10,5-p<0,05\right)$ foram mais frequentes em serviços especializados do que em unidades básicas. Os grupos de adesão $\left(\chi_{2}\right.$ tendência linear $=11,0-p<$ o,oo1) e outras atividades coletivas são mais realizados à medida que aumenta o tamanho do serviço $\left(\chi_{2}\right.$ tendência linear $=17,9-\mathrm{p}<0,001)$.

Apenas 56 serviços $(41,2 \%)$ referiram possuir atividades para populações específicas, sendo a maioria dirigida para gestantes $(38 ; 27,9 \%$, IC $95 \% 20,5-36,3)$.

Como material de apoio às atividades de adesão, a maioria $(68,5 \%)$ utiliza folhetos; $48,6 \%$ referem o uso de frascos de medicamentos. 0 uso de comprimidos avulsos é maior nos serviços especializados $\left(\chi_{2}=15\right.$, - $\mathrm{p}<\mathrm{o}, \mathrm{o1})$ e maiores $\left(\chi_{2}=15,6\right.$ - p < o,o1). Já o emprego de planilhas e de material áudio-visual está associado apenas aos serviços de tamanho médio $\left(\chi_{2}=15,7-p<\right.$ 0,$\left.01 ; \chi^{2}=14,4-\mathrm{p}<0,05\right)$.

Afirmaram ter parcerias estabelecidas com as ONGs 40 serviços (29,4\%). Destes, 23 (57,5\%) encaminham para ONG, $12(30,0)$ têm ONG atuando dentro do serviço e cinco $(12,5 \%)$ utilizam as duas formas de parceria. 0 tamanho e o tipo de serviço possuem associação com a existência de parceria com ONG, que é mais frequente nos ambulatórios especializados em HIV/Aids $\left(\chi_{2}=13,9-p<0,01\right)$ e nos serviços maiores $\left(\chi_{2}=18,4-p<0,01\right)$. Parceria com ONG também está associada ao desenvolvimento de atividades com gestantes $\left(\chi_{2}=5,9-p<0,05\right)$ e portadores de lipodistrofia $\left(\chi_{2}=5,3-p<0,05\right)$.

A análise de correspondências resulta em dois eixos principais que explicam $32 \%$ da variância. 0 mapa fatorial mostra a proximidade de serviços grandes com presença na capital. Os ambulatórios de DST/Aids e de MI apresentam proximidade com estímulo à adesão por psicólogos e assistentes sociais, parcerias com ONGs, fazerem grupos de adesão e outras atividades coletivas (Figura 1). 
Figura I - Mapa fatorial da Análise de Correspondências

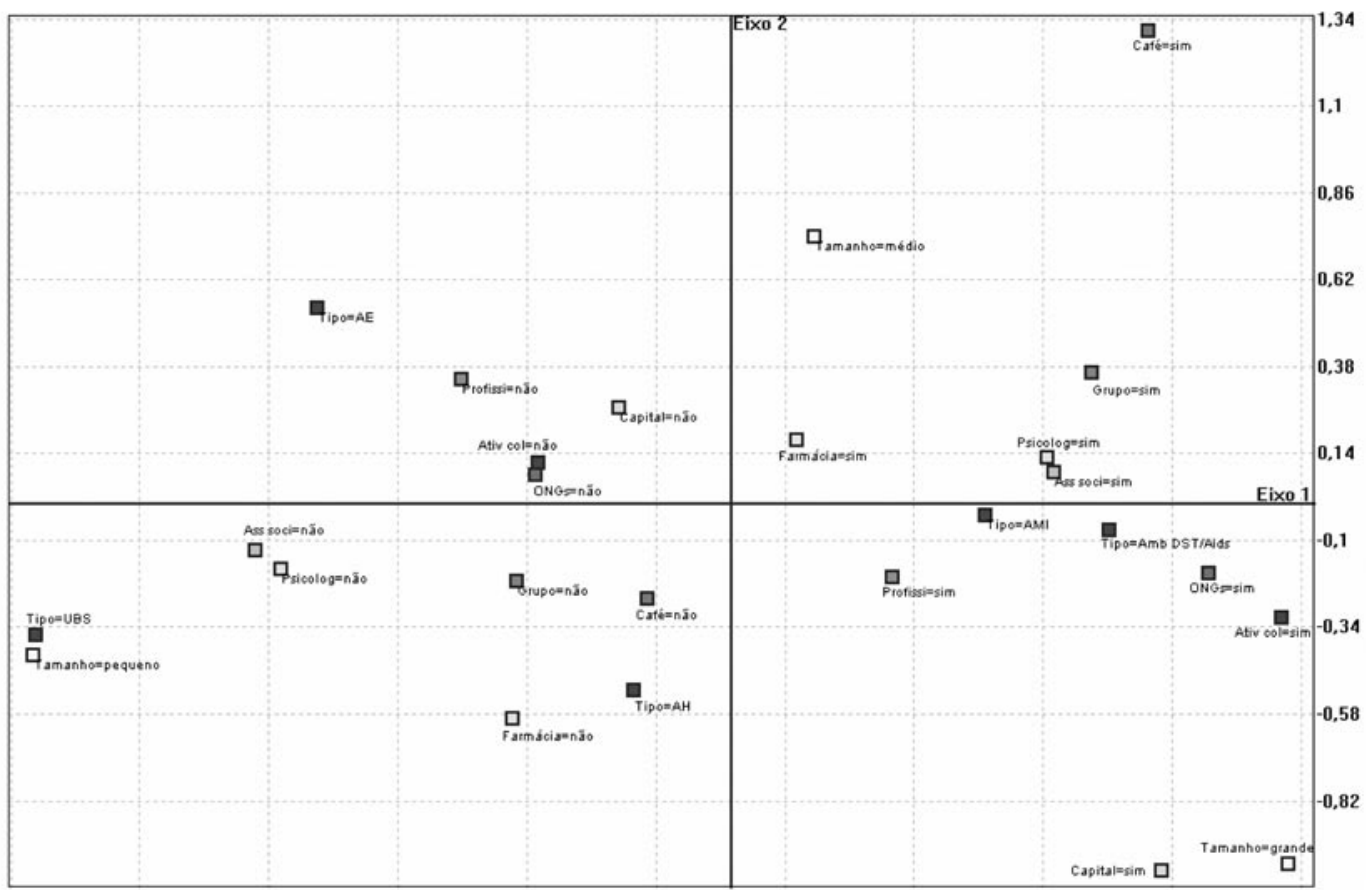

Café - realiza café como atividade coletiva de estímulo à adesão; Grupo - realiza grupo de adesão; Farmácia - avalia adesão através da retirada da medicação na farmácia; Psicolog - consulta do psicólogo como atividade de estímulo à adesão; Ass soci - consulta do Assistente Social como atividade de estímulo à adesão; Profıssi - Cada profissional pergunta de uma maneira para avaliar a adesão; Capital - Serviços localizados na capital; Ativ col - realização de atividades coletivas; ONGs - existência de parceria com ONG; Tipo AE - Ambulatório de Especialidades; Tipo AMI - Ambulatório de Moléstias Infecciosas; Tipo AMB DST/Aids - Ambulatório de DST/Aids; Tipo AH - Ambulatório de Hospital; Tipo UBS - Unidade Básica de Saúde; Tamanho pequeno: até percentil 25; Tamanho médio: percentil 25 a 75; Tamanho grande: maior percentil 75 .

\section{Conclusões}

A adesão à TARV está incorporada às atividades ambulatoriais dos serviços que assistem PVHA no Estado de São Paulo. Quase todos os serviços informam realizar atividades individuais e, a maioria, pelo menos uma atividade coletiva para melhorar a adesão. A maioria dos serviços aborda a adesão nas consultas médicas e de enfermagem. Isso indica que o trabalho de adesão consiste predominantemente de questionamentos e discussões com o paciente. 0 profissional decide de que modo e quando abordar a adesão. Já apontávamos a predominância dessa abordagem inespecífica e altamente dependente do desempenho de cada profissional em estudos conduzidos em 1999 (Castanheira e col., 2000) e 2001 (Melchior e col., 2006).
Os serviços especializados e que acompanham o maior número de pacientes tendem a apresentar atividades mais qualificadas e mais específicas, ou seja, se apoiam mais na equipe multidisciplinar, realizam controle da retirada da medicação na farmácia, possuem mais atividades coletivas e se articulam mais com as ONGs. Isso pode indicar persistência da associação entre melhor qualidade e complexidade do serviço, já apontada em estudo de 2001 (Nemes e col., 2004a). A maior chance de não adesão associada a serviços com menos de 100 pacientes, também já apontada em estudo anterior (Nemes e col., 2004b), ressalta a necessidade de investigar melhor esses aspectos. Adicionalmente, é preciso maior investigação, sobretudo no desenvolvimento e disseminação de tecnologias específicas e potencialmente efetivas para a melhoria da adesão à TARV. 


\section{Referências}

CASTANHEIRA, E. R. L.; CAPOZZOLO, A. A.; NEMES, M. I. B. Características tecnológicas do processo de trabalho em serviços de saúde selecionados. In: BRASIL. Ministério da Saúde. Coordenação Nacional de DST e Aids. Aderência ao tratamento por antiretrovirais em Serviços Públicos no Estado de São Paulo. Brasília: Ministério da Saúde, 2000.

MELCHIOR, R. et al. Avaliação da estrutura organizacional da assistência ambulatorial em HIV/ AIDS no Brasil. Revista de Saúde Pública, São Paulo, v. 4o, n. 1, p. 143-51, 2006.

BRASIL. Ministério da Saúde. Secretaria de Vigilância em Saúde. Programa Nacional de DST e Aids. Monitoraids: Sistema de Monitoramento de Indicadores do Programa Nacional de DST/

Aids. Disponível em: <http://sistemas.aids.gov.br/ monitoraids2/abrir.asp?valor $=234>$. Acesso em: 1 out. 2008.
BRASIL. Ministério da Saúde. Secretaria de Vigilância em Saúde. Programa Nacional de DST e Aids. Diretrizes para o fortalecimento das ações de adesão ao tratamento para pessoas que vivem com HIV e Aids. 2007. Disponível em: <htpp://www.aids. gov.br>. Acesso em: 6 out. 2008.

NEMES, M. I. B.; CARVALHO, H. B.; SOUZA, M. F. M. Antiretroviral therapy adherence in Brazil. AIDS, v. 18, Suppl. 3, p. S15-20, 2004 a.

NEMES, M. I. B. et al. Avaliação da qualidade da assistência no programa de Aids: questões para investigação em serviços de saúde no Brasil. Cadernos de Saúde Pública, Rio de Janeiro, v. 2o, supl .2, p. S310-21, 2004b.

PATERSON, D. L. et al. Adherence to protease inhibitor therapy and outcomes in patients with HIV infection. Annals of Internal Medicine, Philadelphia, v. 133, n. 1, p. 21-30, 2000. 